\title{
Tregs and rethinking cancer immunotherapy
}

\author{
Tyler J. Curiel
}

San Antonio Cancer Institute, University of Texas Health Sciences Center, and Cancer Therapy \& Research Center, San Antonio, Texas, USA.

Tumors express antigens that should induce immune-mediated rejection, but spontaneous rejection of established tumors is rare. Recent work demonstrates that one reason for the lack of tumor rejection is that tumors actively defeat host immunity. This concept forces us to rethink current approaches to harnessing potent, specific host immunity to battle cancer, most of which are based on the paradigm that inducing more antitumor immune cells alone is therapeutic. However, as I discuss in this Personal Perspective, a newer paradigm predicts that reducing tumor-driven immune suppression will be clinically beneficial. $\mathrm{CD} 4^{+} \mathrm{CD} 25^{+}$Tregs are one mechanism of tumor-driven immune evasion that provide prototypical targets for testing novel anticancer treatment strategies within the newer paradigm.

\section{Introduction}

In 2000, Hannahan and Weinberg defined six fundamental hallmarks of cancer (1) that I call the six horsemen of the cancerous apocalypse. These hallmarks are that tumor cells are growth signal self-sufficient, are insensitive to signals that inhibit growth, are able to evade apoptosis, have limitless growth potential, are able to sustain angiogenesis, and are able to invade surrounding tissues and metastasize to distant organs (1). These hallmarks define cancers by focusing on the molecular, biochemical, and cellular traits of the tumor cells. However, it has recently been appreciated that the inability of the immune system to eradicate established tumors is the seventh fundamental hallmark of cancer $(2,3)$.

The scientific rationale behind most strategies to boost the antitumor immune response fails to account fully for this seventh fundamental hallmark. Indeed, tumors employ numerous active mechanisms to suppress host immunity (4). These include altering APC function, fostering dysfunctional $\mathrm{T}$ cell cosignaling, and generating an immune-subversive cytokine milieu. Many of the mechanisms that impede antitumor immunity result in the development of Tregs, the focus of this Personal Perspective. In this article I also discuss how insufficient consideration of the seventh fundamental hallmark of cancer (or the seventh rider of the apocalypse, as I term it), in particular the effect of Tregs, has limited current anticancer immune therapies to only modest successes and points to a new paradigm that is helping to shift thinking about immune-based therapeutic approaches for the treatment of cancer.

\section{Immunologic thinking and infectious disease}

The current paradigm around which most cancer immunotherapies have been developed arose from observations in infections, from which we have derived most of our understanding of immunology. Although immunity to tumors and pathogens share commonalities, they also have substantial differences that fundamentally alter how we might harness immunity to treat these ailments. It is therefore worthwhile to describe briefly how current thinking arose and set the stage for the conceptual shift that is now affording better insights into the immunopathologic basis of cancer. This in turn is leading to the development of novel treatment approaches, including strategies to deal with the effects of Tregs,

Nonstandard abbreviations used: FOXP3, forkhead box P3.

Conflict of interest: The author has declared that no conflict of interest exists.

Citation for this article: J. Clin. Invest. 117:1167-1174 (2007). doi:10.1172/JCI31202. and should ultimately allow optimal use of the awesome potency and specificity of the immune system to treat cancer.

Observations in infections drove early immunologic thinking. Humans attempted to harness the power and exquisite specificity of the immune system to their advantage centuries before Pasteur first demonstrated disease-specific immunity in chickens with cholera (5). However, observations on immunity to cancer were all but absent during this period, probably because of its relative unimportance as a public health threat. It was in 1893 that Coley reported that post-surgical wound erysipelas (an infection with Streptococcus spp.) induced occasional remission of certain cancers (6). This led him to infect cancer patients deliberately with Streptococcus spp., which afforded some therapeutic successes (6). Bacterial LPSinduced TNF- $\alpha$, among other factors, probably contributed to the efficacy of this approach. Nonetheless, more than a century later, cancer immunotherapy still lags substantially behind immunebased approaches for the treatment of infectious diseases.

Immunologic thinking remains grounded in infectious disease principles. By the 1950s, genetically identical mice had been bred at the NIH and elsewhere, allowing for the elegant studies of immunity to influenza that demonstrated that antigen-specific CD8 ${ }^{+}$CTLs induce fully protective immunity, which quickly led to the development of effective human influenza vaccines (7) (although vaccineinduced antibodies are also important for immunity to influenza). These studies established that antigen-specific CTLs were protective against infection, a concept that would quickly spill over into the thinking of tumor immunologists. Demonstration of the critical role of DCs in generating influenza-specific CTLs followed (8), and we now have successful vaccines against other viral infections, including an $\mathrm{HBV}$ vaccine that is based on generating immunity to a single antigen (9). Armed with a centuries-long history of developing successful vaccines to protect individuals against pathogens, investigators attempted to replicate these achievements in cancer, as its public health importance increased.

Goals in tumor immunology aimed to parallel observations in infectious diseases: prove that tumor-specific immunity exists, identify specific tumor-associated antigens, present them on the correct APCs (especially DCs), and induce curative tumor antigen-specific $\mathrm{CD}^{+}$CTLs. Following this reasoning, Prehn and colleagues demonstrated tumor-specific immunity in mice using distinct chemically induced sarcomas half a century ago (10). Over the following two decades, many tumor antigens were identified (11) and shown to elicit antigen-specific antitumor $\mathrm{CD}^{+}$CTLs (12). Definition of an important role for DCs in initiating antitumor immunity 


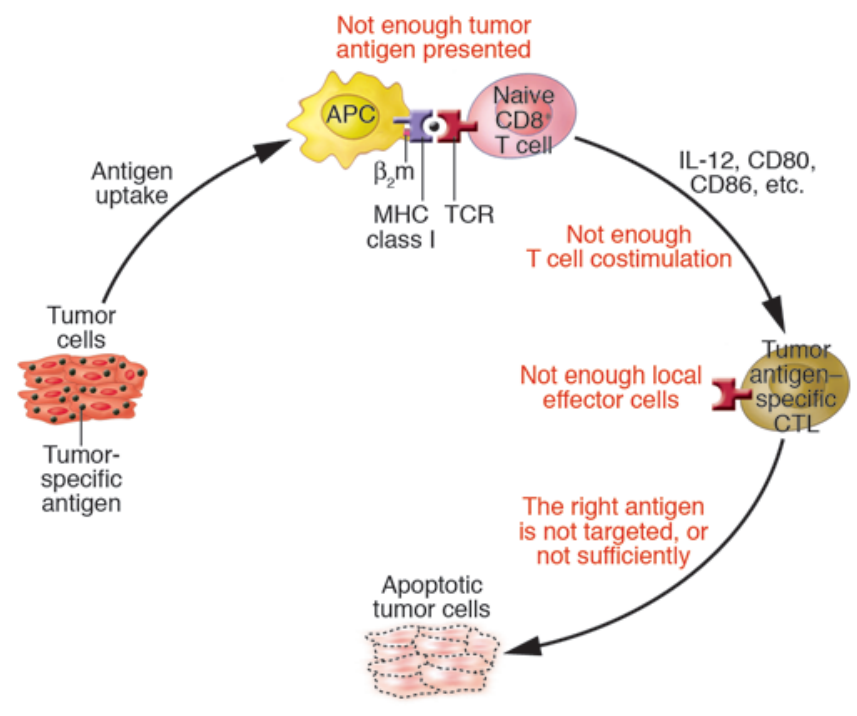

Figure 1

The not enough of a good thing paradigm. APCs (especially DCs) capture antigen from tumor cells. Antigen-loaded APCs then prime naive $T$ cells to become antitumor CTLs through soluble mediators such as IL-12 and/or cosignaling molecules such as CD80 or CD86. Failure to elicit protective immunity is seen as a lack of sufficient quantity or quality of 1 of these events (indicated in red text). This model predicts that augmenting numbers or quality of a specific component will suffice as a clinical strategy.

followed (13). Discoveries unfolded as expected, setting the stage for the current paradigm that drives most thinking regarding the development of immune-based strategies to treat cancer.

\section{The current paradigm}

Figure 1 outlines the current paradigm in tumor immunity: tumors express tumor-associated antigens that can be captured by professional APCs, notably DCs, which prime naive T cells (through the expression of cosignaling molecules, the production of soluble factors, and other mechanisms) to become antigen-specific CD8 ${ }^{+}$CTLs. These CTLs, when in sufficient abundance and directed against the appropriate tumor antigens, then eradicate the tumor. According to this paradigm, in order to engender curative antitumor immunity one must supply more antigen or the appropriate antigen, augment the effects of the cosignaling molecules and/or soluble factors that induce the generation of antigenspecific $\mathrm{CD}^{+}$CTLs, or boost deficient numbers of APCs and/or antigen-specific $\mathrm{T}$ cells. In other words, there is just not enough of a good thing (e.g., antigens, priming or activating signals, APCs, or effector cells), and simply supplying more of the missing elements should result in immune rejection of the tumor.

On the basis of this paradigm, numerous anticancer immunotherapeutic strategies have been developed. These include infusing additional tumor antigen or antigen-pulsed APCs (14-16); supplying $\mathrm{T}$ cells generated from tumor-infiltrating lymphocytes (17), $\mathrm{T}$ cells together with a soluble growth factor (18), T cells activated ex vivo with cytokines (19), or T cells engineered to express receptors for specific tumor-associated antigens (20); and boosting the effect of cosignaling molecules or activating cytokines. Research strategies have included identifying novel tumor-associated antigens (21). Although these approaches are generally sound and well exe- cuted, they ignore the important reality that many patients harbor abundant tumor antigens, DCs, and antigen-specific effector cells but do not eradicate their tumors $(3,22)$. Hence, simply boosting numbers of existing troops might not suffice to win this war. A further complication is that many approaches are logistically difficult, costly, labor and time intensive, subject to substantial regulatory and safety concerns, and applicable to only a minority of patients, in addition to being relatively ineffective by standard measures of clinical success (23). Therefore, it is now becoming clear that current immune-based approaches to cancer therapy require substantial rethinking if they are to realize the same potential as those used in defense against pathogens.

\section{Problems with the current paradigm}

Infections are pathologic inflammatory processes driven by rapidly dividing cells or by virions of extrinsic origin. Conversely, cancers are pathologic inflammatory processes driven by rapidly dividing cells of intrinsic (self) origin, and therein lies the fundamental difference between infectious diseases and cancer. Because tumors are intrinsic, generating an effective antitumor immune response requires mounting a substantial autoimmune attack, which involves breaking self tolerance (the mechanisms that prevent the immune system from attacking one's own tissues). Of course, not all tumor antigens are self antigens (e.g., EBV antigens in some lymphomas and mutated p53 in many epithelial carcinomas; refs. 24, 25). Nonetheless, self tolerance is a large obstacle to antitumor immunity that is not usually germane to discussions of antipathogen defenses. The important mouse model of infection with influenza taught us a great deal about protective immunity as it relates to APCs, protective antigens, and specific CTLs, but says nothing regarding breaking self tolerance. This issue has only recently begun to receive serious mainstream consideration in tumor immunotherapy $(26,27)$.

Furthermore, it has become clear that inefficient tumor rejection is not just passive, a result of insufficient effector cells $(22,28)$. Recent discoveries show that tumors actively fight back by producing immunosuppressive factors such as IL-10 (29-31), TGF- $\beta$ $(22,32-34)$, and VEGF (35). These factors and other immunosuppressive mechanisms induced by the tumor are likely to arise, as efforts by the immune system to purge abnormal tumor tissue simultaneously elicit intrinsic mechanisms that protect against the autoimmunity engendered by most antitumor immune responses. Therefore, the fundamental problem in tumor immunity is not that there is not enough of a good thing, but rather that there is too much of a bad thing. This important shift in thinking has not figured substantially in the development of current tumor immunotherapy strategies.

\section{Tregs}

The advantage of the vast diversity of $\mathrm{T}$ cell and $\mathrm{B}$ cell receptors created by random genetic recombination (36-38) is that they have the potential to recognize an essentially infinite number of antigens. Nonetheless, because the process is random it also produces immune cells bearing receptors that recognize self antigens (i.e., autoreactive cells). An important mechanism of defense against autoimmunity is the permanent deletion of autoreactive $T$ cells in the fetal thymus (39). However, self-reactive T cells escaping thymic deletion and seeding the peripheral lymphoid organs require another strategy to curb their autoimmune potential (40). A suppressor $\mathrm{T}$ cell blocking peripheral autoreactive $\mathrm{T}$ cells was identified about 40 years ago, but progress was hampered by a lack of 


\section{A Thymus}

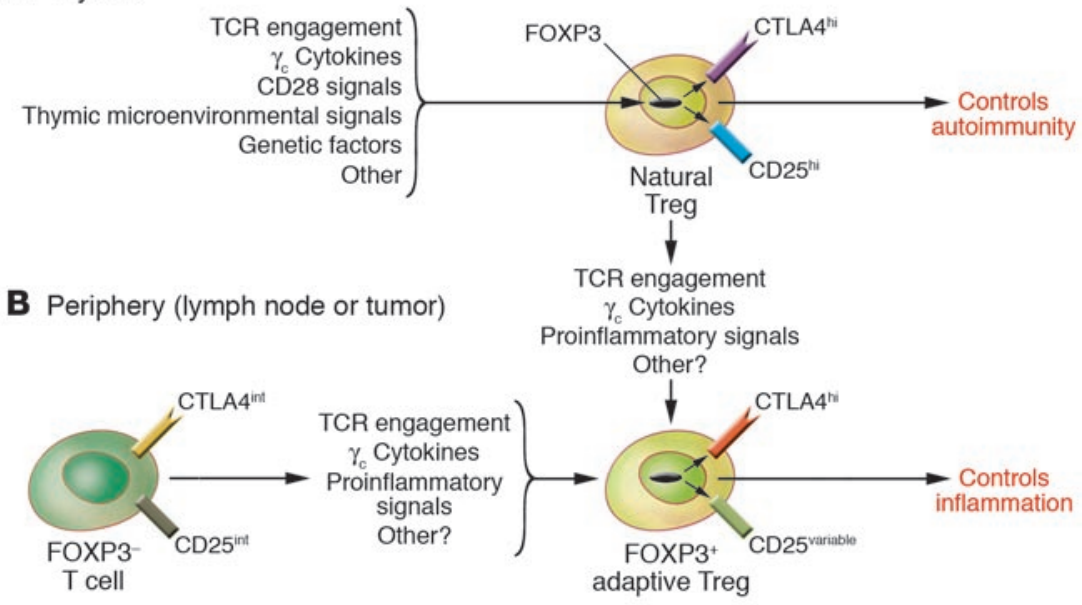

\section{Figure 2}

Natural and adaptive Tregs. (A) Natural Tregs arise in the thymus under homeostatic conditions, through the integration of a myriad of local thymic factors. Thymically derived natural Tregs defend against autoimmunity. (B) Adaptive Tregs arise in the periphery (outside the thymus), either from natural Tregs receiving additional signals or through an independent pathway. Both natural and adaptive Tregs might contribute to tumor immunopathology. CTLA4, cytotoxic T lymphocyte-associated antigen 4. specific techniques to identify the cells and because of scientific skepticism regarding suppression. However, Sakaguchi and colleagues resurrected interest in suppressor cells in 1995 with an influential paper demonstrating that suppressive function largely resided in a population of $\mathrm{CD} 4^{+} \mathrm{T}$ cells expressing high levels of CD25 (41), what we now call CD $4^{+} \mathrm{CD} 25^{+}$Tregs. Tregs suppress autoreactive $T$ cells without killing them $(42,43)$ through incompletely understood, contact-dependent mechanisms (40, 42-47).

Suppression of tumor immunity by CD $4^{+}$Tregs, which was originally described in the early 1980s (48), was largely ignored. However, the demonstration that Treg depletion in mouse models of cancer improves endogenous immune-mediated tumor rejection (49) and tumor antigen-specific immunity (50) quickly rekindled interest in the role of Tregs in tumor immunopathology. Further work demonstrated that Treg depletion augments tumor immunotherapy, including vaccination (51) and cytotoxic T lymphocyte-associated antigen 4 (CTLA4) blockade (52), and even augments immunologic rejection of brain tumors (53), which are notoriously difficult to treat using immunotherapeutic approaches because of the blood-brain barrier. Tregs can inhibit tumor-specific $\mathrm{CD}^{+}(54)$ and $\mathrm{CD}^{+}(55) \mathrm{T}$ cell effector functions through incompletely understood mechanisms including cell-cell contact and/or the production of soluble factors such as IL-10 or TGF- $\beta$ $(42,43,54,56-58)$. Numbers of Tregs are increased in the blood $(42,43,59-66)$ and populate the tumor mass and draining lymph nodes $(60,67-72)$ of patients with many different cancers.

An important issue is why the number of Tregs is increased in individuals with cancer. Are the cells attempting to suppress antitumor immunity sensed as an autoreactive immune response, are they responding to the inflammation, or is there something else? That is, is the tumor using Tregs to orchestrate its own defense against the constant assault of host immune surveillance? In that case, the Tregs could increase in number if the host senses antitumor immunity as pathologic autoimmunity and deploys them in misguided efforts to quash rebellious anti-self (i.e., antitumor) immune cells. Teleologically, the tumor could be orchestrating its own defense against the constant assault of host immune surveillance using Tregs as potent contrivances of convenience.

In order to answer these questions, it is important to understand that there are two phenotypically identical populations of $\mathrm{CD} 4{ }^{+} \mathrm{CD} 25^{+}$Tregs - natural and adaptive (73). Natural
$\mathrm{CD} 4^{+} \mathrm{CD} 25^{+}$Tregs arise in the thymus under homeostatic conditions to safeguard against autoimmunity. Adaptive $\mathrm{CD} 4^{+} \mathrm{CD} 25^{+}$ Tregs arise during inflammatory processes such as infections and cancers (Figure 2) and suppress immunity through heterogeneous mechanisms that include direct contact or the production of soluble factors such as IL-10 and TGF- $\beta$ (73). Tregs that infiltrate the tumor microenvironment are probably adaptive, although it is not clear whether they differentiate from natural Tregs, as suggested in a recent report (34), or are induced from another precursor cell (Figure 2B). The tumor itself (34) and cells in the tumor microenvironment, such as DCs, induce the differentiation of Tregs through various mechanisms including the production of TGF- $\beta(34,74)$ and the expression of B7-H1 (75). Different pathways of Treg differentiation in tumors probably lead to heterogeneous populations of infiltrating Tregs and might account for conflicting reports regarding their mechanisms of suppression and other attributes $(42,43,76)$. At least some tumor-associated Tregs are specific for tumor antigens (77), although once activated, they can also suppress tumor antigen-independent immune responses though bystander mechanisms. In addition to inhibiting the function of $\mathrm{T}$ cells, Tregs also inhibit the function of NK cells (78), B cells (79), and other immune cells. Several comprehensive reviews of tumor-associated Tregs provide additional details of the cells suppressed and the mechanisms of suppression $(42,43,76)$.

An important issue is the inability to identify individual functional Tregs easily. Only a subset of the $\mathrm{T}$ cells identified as $\mathrm{CD} 4^{+} \mathrm{CD} 25^{+}$by flow cytometry are functionally suppressive Tregs. The remainder are mostly activated effector $\mathrm{T}$ cells $(42,43,76)$. Confining analysis to $\mathrm{CD}^{+} \mathrm{T}$ cells expressing high levels of CD25 increases the proportion that are functionally suppressive Tregs, but this is still not absolutely diagnostic, nor is the inclusion of additional cell surface molecules such as CTLA4, glucocorticoid-induced TNF receptor-related protein (GITR), and CD103, as all these are also expressed by activated effector $\mathrm{CD}^{+}{ }^{+} \mathrm{T}$ cells $(40,42-47,76)$.

More recently, expression of the nuclear transcription factor forkhead box P3 (FOXP3), which is critical for the development of natural $\mathrm{CD}^{+} \mathrm{CD} 25^{+}$Tregs $(80-82)$, has been used as a surrogate for Tregs in both mice and humans (83). Nonetheless, the function of $\mathrm{CD} 4^{+} \mathrm{CD} 25^{+} \mathrm{FOXP} 3^{+} \mathrm{T}$ cells in a given setting must be 


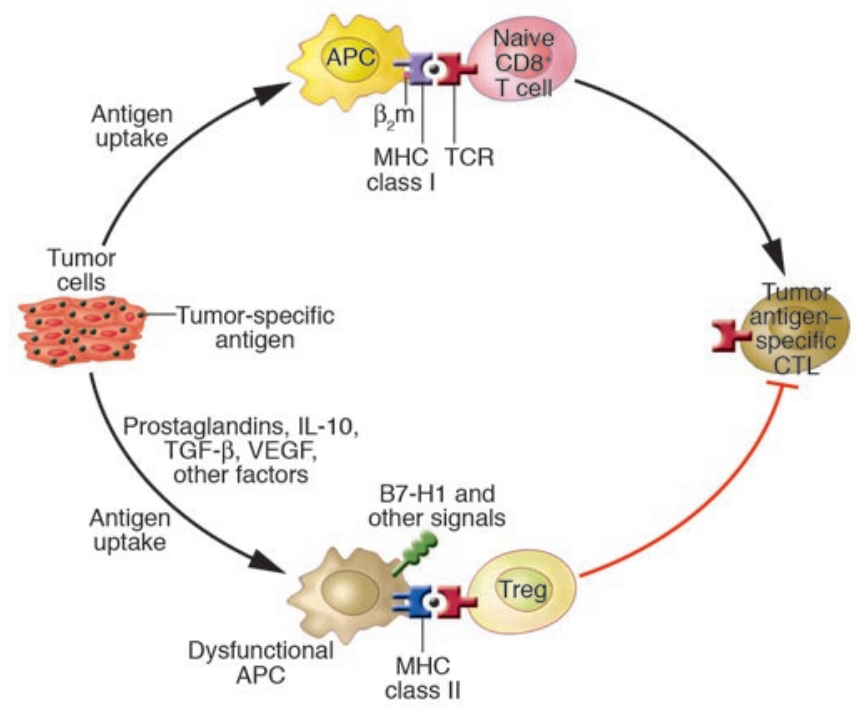

Figure 3

The too much of a bad thing paradigm. Dysfunctional APCs, aberrant cosignaling, and other factors (such as prostaglandins, counterregulatory cytokines, and hypoxia) help generate Tregs and other mediators of immune pathology. These pathways, and the products and cells that they generate, must be defeated for optimal tumor immunotherapy.

confirmed, because it is now clear that some FOXP3- $\mathrm{T}$ cells are suppressive and that not all FOXP3 ${ }^{+}$cells are Tregs $(46,47,82)$. In this regard, it is important to reevaluate prior work on Tregs defined as $\mathrm{CD} 4^{+} \mathrm{CD} 25^{+} \mathrm{T}$ cells without regard to FOXP3 status. Antibodies specific for FOXP3 (84) and mice expressing reporter genes under the control of Foxp3 regulatory elements $(85,86)$ have spawned an explosion of new findings in the past few years, allowing additional definitive studies of Tregs $(46,47)$, although the inability to isolate viable FOXP3 ${ }^{+}$human cells for functional studies continues to provide considerable ambiguities and difficulties. Further work is likely to yield additional means of identifying functional Tregs, providing deeper insights and helping to resolve contradictory results regarding their origins and suppressive mechanisms. For example, low expression levels of phosphodiesterase $3 \mathrm{~B}$ has just been proposed as a definitive signature of natural $\mathrm{CD} 4^{+} \mathrm{CD} 25^{+}$Tregs (46).

\section{Immune dysfunction in ovarian cancer as a model for the too much of a bad thing paradigm}

Studying individuals with ovarian cancer has provided my group with an opportunity to determine why their tumors are not cleared immunologically despite the presence of ample tumorspecific immunity $(60,87)$. I selected patients with ovarian cancer because many such individuals develop tumor-derived fluid in the abdomen (i.e., malignant ascites) that contains large quantities of tumor antigens, other soluble microenvironmental factors, and immune cells. Furthermore, tumors from individual patients can be grown for analysis of tumor-specific immunity using autologous immune cells $(75,88)$, a rarity in human studies. My colleagues and I showed that in the ovarian cancer microenvironment, plasmacytoid DCs ( $\mathrm{pDCs}$ ) induced T cells to produce the immunosuppressive cytokine IL-10 - suggestive of adaptive Tregs - rather than protective IFN- $\gamma$, as did pDCs from normal individuals (88). We further showed that myeloid DCs, long used as vehicles for cancer vaccines (16), were conditioned by the tumor microenvironment to express the cosignaling molecule B7-H1, which induces T cells to produce IL-10 (75), again suggestive of an adaptive Treg phenotype.

We then demonstrated that Tregs actively accumulated in the human ovarian cancer microenvironment, inhibiting tumorspecific cytotoxicity and cytokine production by tumor-specific $\mathrm{CD}^{+} \mathrm{T}$ cells in vitro and in vivo (60). Importantly, there was an inverse correlation between the number of Tregs in the tumor and patient survival (60), which was corroborated independently by the demonstration that high levels of FOXP3 in the tumor microenvironment predicted reduced survival in patients with ovarian cancer (89).

Recent reports show that the number of $\mathrm{CD} 4^{+} \mathrm{CD} 25^{+} \mathrm{FOXP} 3^{+}$ $\mathrm{T}$ cells correlates inversely with clinical outcomes in several epithelial carcinomas, including ovarian cancer (60), breast cancer (90), and hepatocellular carcinoma (91). However, the situation in hematologic malignancies differs. Increased numbers of tumor-infiltrating $\mathrm{FOXP}^{+} \mathrm{T}$ cells predicts improved survival in individuals with follicular lymphoma (70), and reduced numbers of FOXP3 ${ }^{+}$cells predicts poor survival in individuals with Hodgkin lymphoma (71). However, in these studies, the function of the FOXP3 $3^{+}$cells was not tested, and not all FOXP3 ${ }^{+} \mathrm{T}$ cells are functionally suppressive $(46,47,82)$. In contrast, both the number and the function of FOXP3 ${ }^{+}$Tregs was reduced in patients with multiple myeloma or monoclonal gammopathy of uncertain significance (92), and Treg function decreased as tumor burden increased in a small series of patients with cutaneous T cell leukemia/lymphoma (72). Therefore, it is possible that the effects of Tregs or the differentiation of FOXP3 ${ }^{+}$cells into functional Tregs fundamentally differs in lymphoid malignancies compared with epithelial carcinomas. Alternatively, it is possible that the appropriate $\mathrm{T}$ cell compartment was not studied in the context of lymphoid malignancies. The utility of FOXP3 content and Treg function as prognostic factors in different cancers is an area of intense investigation, but much remains to be understood.

\section{Potential clinical applications}

Tregs in the tumor microenvironment are common mediators of distinct active immune evasion mechanisms in the too much of a bad thing paradigm (Figure 3). Therefore, reducing Treg function in cancer patients could be therapeutic, analogous to the benefits seen in mouse models (49-52). For rapid clinical translation of this concept, denileukin diftitox (Ontak) has been considered. Denileukin diftitox is a recombinant fusion protein consisting of IL-2 and diphtheria toxin that has been approved by the FDA to treat cutaneous $\mathrm{T}$ cell leukemia/lymphoma, which is characterized by large numbers of malignant $\mathrm{CD} 4^{+} \mathrm{CD} 25^{+} \mathrm{T}$ cells $(72,93)$.

CD25 is the IL-2 receptor $\alpha$ chain. Denileukin diftitox binds the IL-2 receptor and inhibits protein translation following internalization, leading to apoptosis (93). Based on phenotypic similarities between $\mathrm{CD} 4{ }^{+} \mathrm{CD} 25^{+}$malignant leukemic cells and Tregs, we undertook a phase $0 /$ I clinical trial enrolling patients with any advanced-stage epithelial carcinoma to test the hypothesis that denileukin diftitox would deplete Tregs, thereby reducing immune suppression and boosting antitumor immunity (94). Our data suggested that denileukin diftitox at 9 or $12 \mu \mathrm{g} / \mathrm{kg}$ decreased the number of blood Tregs and the suppression mediated by the 
$\mathrm{CD} 4^{+} \mathrm{CD} 25^{+}$blood $\mathrm{T}$ cell population in patients with ovarian, lung, breast, or pancreatic cancer for up to 30 days after a single infusion, in association with improved cellular immunity, and was well tolerated. We therefore propose that the decreased number of Tregs enabled immune-mediated tumor clearance and are currently investigating this possibility. A recent report supported this idea by demonstrating that denileukin diftitox reduced Treg numbers and overall suppression mediated by the $\mathrm{CD} 4{ }^{+} \mathrm{CD} 25^{+}$ cell population in a mouse model of breast cancer, with improved immunity and tumor regression (95).

Additional reports confirm that denileukin diftitox depletes the number of Tregs and the suppression mediated by the $\mathrm{CD} 4^{+} \mathrm{CD} 25^{+}$ $\mathrm{T}$ cell population and improves immunity in individuals with renal cell carcinoma $(96)$ and melanoma $(97,98)$. However, one group demonstrated no reduction in the number of Tregs or their function in melanoma patients receiving denileukin diftitox (99), although the reasons for these conflicting results are unknown. We are conducting an ongoing phase II efficacy trial of denileukin diftitox for patients with stage III or IV epithelial ovarian carcinoma that are failing standard treatment (identifier NCT00238186; http://www.clinicaltrials.gov) that is designed to address and resolve some of these issues.

It is now clear that Treg function is compartmentalized. For example, in a mouse model of metastatic colon cancer, functional $\mathrm{CD}^{+}$Tregs preferentially developed in the lung (100). In another mouse model of cancer, depletion of intratumoral Tregs enhanced adoptive $\mathrm{T}$ cell immunotherapy (69). In human breast cancer, tumor-bearing lymph nodes expressed higher FOXP3 mRNA than did tumor-negative lymph nodes (67). Some B cell lymphomas recruit functional Tregs into the tumor through CC chemokine ligand 22 (CCL22) produced by the tumor cells (68), as has also been shown in ovarian carcinoma (60). Furthermore, Tregs were abundant in the solid tumor and malignant ascites in ovarian cancer, but rare in local lymph nodes (60). Therefore, it is important to study Tregs in the appropriate anatomical compartment, and it is likely necessary to deplete them locally in the tumor or draining lymph nodes (where $\mathrm{T}$ cell priming occurs) for optimal therapeutic utility. The relationship between Treg numbers and function in the blood - an easily accessible compartment for study - versus the tumor is largely unknown and an area ripe for further study.

Treg depletion is one avenue in which to test the too much of a bad thing paradigm, and denileukin diftitox is one agent for this purpose. However, we have identified numerous additional agents and strategies (101), including blocking Treg trafficking, differentiation, and/or function and reducing effector cell susceptibility to suppression; some of these have already proven successful in preliminary studies $(60,102)$.

\section{Tregs might account for the failure of some cancer immunotherapies}

The too much of a bad thing paradigm offers novel insights into the failure of current immunotherapy strategies. For example, active vaccination of patients with cancer can induce antigen-specific Tregs (103). This result should not be surprising, as Tregs are simply another type of antigen-specific $\mathrm{T}$ cell elicited during an immune response. Prior failure to identify vaccine-induced Tregs simply reflects a failure to look. It is probable that antigen-specific Tregs are elicited by many active cancer vaccines. Further investigation of how they ultimately influence vaccine-induced immunity is therefore needed. For instance, the clinical responses induced by some vaccines could be a result of rapid induction of antigenspecific CTLs that overwhelm the deleterious effects of simultaneously generated Tregs. However, tumors might later recur or progress as these CTLs undergo programmed cell death and disappear, allowing eventual domination by the longer-lived Tregs. Similarly, DC vaccines might produce clinical responses early but not durably (16), because although the induced CTLs initially combat the tumor, they then fail as DC-induced Tregs dominate. The recent demonstration that infused DCs induced Tregs in patients with myeloma supports this concept (104).

IL-2 expands and activates CTLs (19), which might explain early clinical responses in patients with melanoma and renal cell carcinoma treated with that cytokine. However, IL-2 also boosts human Treg function in vivo (105), which can ultimately inhibit antitumor CTLs, explaining the low clinical response rate with IL-2 treatment. IFN- $\gamma$, now in human cancer trials for ovarian cancer $(106,107)$, can inhibit DC differentiation (108) and induce DC expression of B7-H1 (75), both of which induce Treg differentiation.

These studies suggest that the induction or presence of Tregs might account for the failure of some cancer immunotherapies. Therefore, consistent with the concept that most effective cancer therapies are multimodal, combining Treg depletion with active cancer immunotherapeutic interventions is an attractive prospect, supported by abundant data in mice $(51-53,69)$ and by preliminary human trials $(95,96,98)$. Furthermore, the not enough of a good thing paradigm explains many important features of tumors, such as ineffective antigen presentation and poor $\mathrm{T}$ cell activation (27). These features could defeat strategies to deplete Tregs as the sole intervention.

However, immunologic responses are highly interrelated and complex. Therefore, combining Treg depletion with additional modalities requires much additional investigation. For example, depleting Tregs too early before or at any time after vaccination in a mouse model for multimodal cancer therapy enormously reduced the capacity of Treg depletion to boost immunity compared with that observed when Tregs were depleted at vaccination, the optimal time point (109). Combining chemotherapy with immunotherapy is an additional approach likely to be enhanced by Treg depletion (110). Vaccinating against FOXP3 itself augments tumor vaccines (111), although the practical utility of this approach is uncertain because depleting all FOXP3 $3^{+}$Tregs could promote pathologic autoimmunity. Of course, only clinical trials will ultimately determine whether net effects of specific approaches are beneficial or deleterious.

Immunopathologic contributions of other regulatory cell populations such as CD8 ${ }^{+}$Tregs $(100,112)$, NKT cells (113), myeloidderived suppressor cells $(114,115)$, and B7-H4+ myeloid cells (116) remain to be fully established and merit further investigation. For example, in some mouse models for cancer, depleting regulatory NKT cells improves tumor immunity, whereas depleting $\mathrm{CD} 4{ }^{+} \mathrm{CD} 25^{+}$Tregs does not (117).

To a hammer, the world looks like a nail. I could thus be accused of seeing Tregs lurking around every corner. Nonetheless, as we come to appreciate the complexities of tumor immunity, we are likely to view modes of action of some agents differently. VEGF (102), prostaglandins (118), and estrogen (119) probably contribute to Treg differentiation and/or function in some tumors. Agents blocking these signals might therefore be therapeutically useful 
through Treg depletion in addition to their known mechanisms. For instance, blocking VEGF reduced the number of intratumoral Tregs in mouse models of colon cancer and melanoma (102), and cyclophosphamide, a standard cytotoxic chemotherapeutic (120), has long been known to inhibit suppressor T cells (121), which is now recognized as a result of Treg depletion (122).

\section{Remaining issues}

Because we are so early into our journey of discovery in this new landscape of immunologic thinking, it is practically ludicrous to catalog what remains to be discovered, because it is almost everything. Nonetheless, here I pose some of the important remaining questions. What local factors in the tumor microenvironment augment expansion of the Treg population or favor survival of Tregs over antitumor effector cells? From which precursor populations do Tregs in the tumor arise? What is the antigen specificity of Tregs; does it differ from effector immunity; and does deletion of antigen-specific Tregs produce immunologic consequences different from those of strategies that deplete all Tregs? Which regulatory cell population, if any, has the most immunopathologic importance in a given cancer? Practical questions include: Do agents used to reduce Treg function work equally well in the blood, tumor, and draining lymph nodes? What is the relationship between Treg depletion in the blood and at local sites, and do such observations predict immunologic or clinical outcomes?

\section{Concluding remarks}

Immune-based therapy arguably remains the best option to cure many types of cancer. There is simply nothing as potent or tar- geted as antigen-specific immunity. The not enough of a good thing paradigm has helped to elucidate important principles regarding the use of immunotherapy to treat cancer. Nonetheless, in my opinion, its conceptual basis prevents its optimal use.

Lack of immune rejection is the seventh fundamental hallmark of cancer. Additional tumor-mediated mechanisms defeating host immunity are known or surely will be discovered that will lead to many more approaches within this paradigm. As tumor immunologists shake off the shackles of pathogen-based dogma that have impeded progress in developing effective cancer immunotherapy, we might at last see this approach reach its zenith. Just as a prudent general only reluctantly sends troops into battle across a known mine field without first sending in mine sweepers, the too much of a bad thing paradigm suggests that to optimize currently employed immunotherapeutic strategies, the malignant equivalent of the mine field must first be swept as well.

\section{Acknowledgments}

I thank the many members of my research team for invaluable scientific contributions. The author is supported by NIH grants CA100425 and CA105207, by FDA grant FD003118, and by the Fanny Rippel Foundation.

Note: Due to space constraints, a number of important references could not be included.

Address correspondence to: Tyler J. Curiel, San Antonio Cancer Institute, University of Texas Health Sciences Center, 7703 Floyd Curl Drive, San Antonio, Texas 78229-3900, USA. Phone: (210) 562-5286; Fax: (210) 562-5292; E-mail: curielt@uthscsa.edu.
1. Hanahan, D., and Weinberg, R.A. 2000. The hallmarks of cancer. Cell. 100:57-70.

2. Dunn, G.P., Old, L.J., and Schreiber, R.D. 2004 The three Es of cancer immunoediting. Annu. Rev. Immunol. 22:329-360.

3. Zitvogel, L., Tesniere, A., and Kroemer, G. 2006 Cancer despite immunosurveillance: immunoselection and immunosubversion. Nat. Rev. Immunol. 6:715-727.

4. Zou, W. 2005. Immunosuppressive networks in the tumour environment and their therapeutic relevance. Nat. Rev. Cancer. 5:263-274.

5. Silverstein, A.M. 1989. A history of immunology. Academic Press. San Diego, California, USA. 422 pp.

6. Coley, W.B. 1893. The treatment of malignant tumors by repeated inoculums of erysipelas. Am.J. Med. Sci. 105:487-511.

7. McMichael, A.J., Gotch, F.M., Noble, G.R., and Beare, P.A. 1983. Cytotoxic T-cell immunity to influenza. N. Engl.J. Med. 309:13-17.

8. Nonacs, R., Humborg, C., Tam, J.P., and Steinman, R.M. 1992. Mechanisms of mouse spleen dendritic cell function in the generation of influenza-specific, cytolytic T lymphocytes. J. Exp. Med. 176:519-529.

9. Crosnier, J., et al. 1981. Randomised placebo-controlled trial of hepatitis B surface antigen vaccine in french haemodialysis units: II, haemodialysis patients. Lancet. 1:797-800.

10. Prehn, R., and Main, J.M. 1957. Immunity to methylcholanthrene-induced sarcomas. J. Natl. Cancer Inst. 18:769-778.

11. Schreiber, H., Ward, P.L., Rowley, D.A., and Stauss, H.J. 1988. Unique tumor-specific antigens. Annu. Rev. Immunol. 6:465-483.

12. Stauss, H.J., Van Waes, C., Fink, M.A., Starr, B., and Schreiber, H. 1986. Identification of a unique tumor antigen as rejection antigen by molecular cloning and gene transfer. J. Exp. Med. 164:1516-1530.
13. Banchereau, J., and Steinman, R.M. 1998. Dendritic cells and the control of immunity. Nature. 392:245-252

14. Hsu, F.J., et al. 1996. Vaccination of patients with B-cell lymphoma using autologous antigen-pulsed dendritic cells. Nat. Med. 2:52-58.

15. Nestle, F.O., et al. 1998. Vaccination of melanoma patients with peptide- or tumor lysate-pulsed dendritic cells. Nat. Med. 4:328-332.

16. Nestle, F.O., Banchereau, J., and Hart, D. 2001. Dendritic cells: on the move from bench to bedside. Nat. Med. 7:761-765.

17. Rosenberg, S.A., Spiess, P., and Lafreniere, R. 1986. A new approach to the adoptive immunotherapy of cancer with tumor-infiltrating lymphocytes. Science. 233:1318-1321.

18. Rosenberg, S.A., et al. 1988. Use of tumor-infiltrating lymphocytes and interleukin- 2 in the immunotherapy of patients with metastatic melanoma. A preliminary report. N. Engl. J. Med. 319:1676-1680.

19. Rosenberg, S.A., et al. 1987. A progress report on the treatment of 157 patients with advanced cancer using lymphokine-activated killer cells and interleukin-2 or high-dose interleukin-2 alone. N. Engl. J. Med. 316:889-897.

20. Morgan, R.A., et al. 2006. Cancer regression in patients after transfer of genetically engineered lymphocytes. Science. 314:126-129.

21. Pardoll, D.M. 1998. Cancer vaccines. Nat. Med. 4:525-531.

22. Gajewski, T.F., Meng, Y., and Harlin, H. 2006. Immune suppression in the tumor microenvironment. J. Immunother. 29:233-240.

23. Curiel, T.J., and Curiel, D.T. 2002. Tumor immunotherapy: inching toward the finish line. J. Clin. Invest. 109:311-312. doi:10.1172/JCI200215042.

24. Spiotto, M.T., Fu, Y.X., and Schreiber, H. 2003. Tumor immunity meets autoimmunity: antigen levels and dendritic cell maturation. Curr. Opin. Immunol. 15:725-730.

25. Smyth, M.J., Dunn, G.P., and Schreiber, R.D. 2006 Cancer immunosurveillance and immunoediting: the roles of immunity in suppressing tumor development and shaping tumor immunogenicity. $A d v$. Immunol. 90:1-50.

26. Pardoll, D. 2001. T cells and tumours. Nature. 411:1010-1012.

27. Pardoll, D. 2003. Does the immune system see tumors as foreign or self? Annu. Rev. Immunol. 21:807-839.

28. Wick, M., et al. 1997. Antigenic cancer cells grow progressively in immune hosts without evidence for T cell exhaustion or systemic anergy. J. Exp. Med. 186:229-238.

29. Elgert, K.D., Alleva, D.G., and Mullins, D.W. 1998. Tumor-induced immune dysfunction: the macrophage connection. J. Leukoc. Biol. 64:275-290.

30. Salazar-Onfray, F. 1999. Interleukin-10: a cytokine used by tumors to escape immunosurveillance. Med. Oncol. 16:86-94.

31. Moore, K.W., de Waal Malefyt, R., Coffman, R.L., and O'Garra, A. 2001. Interleukin-10 and the interleukin-10 receptor. Annu. Rev. Immunol. 19:683-765.

32. Li, M.O., Wan, Y.Y., Sanjabi, S., Robertson, A.K., and Flavell, R.A. 2006. Transforming growth factor-beta regulation of immune responses. Annu. Rev. Immunol. 24:99-146.

33. Saunier, E.F., and Akhurst, R.J. 2006. TGF beta inhibition for cancer therapy. Curr. Cancer Drug Targets. 6:565-578.

34. Liu, V.C., et al. 2007. Tumor evasion of the immune system by converting CD4+CD25- T cells into CD4+CD25+ T regulatory cells: role of tumorderived TGF-beta. J. Immunol. 178:2883-2892.

35. Gabrilovich, D.I., et al. 1996. Production of vas- 


\section{review series personal perspective}

cular endothelial growth factor by human tumors inhibits the functional maturation of dendritic cells. Nat. Med. 2:1096-1103.

36. Palmer, E. 2006. The T-cell antigen receptor: a logical response to an unknown ligand. J. Recept. Signal Transduct. Res. 26:367-378.

37. Roth, D.B. 2003. Restraining the V(D)J recombinase. Nat. Rev. Immunol. 3:656-666.

38. Krangel, M.S. 2003. Gene segment selection in V(D)J recombination: accessibility and beyond. Nat. Immunol. 4:624-630.

39. Sprent, J., and Surh, C.D. 2003. Knowing one's self: central tolerance revisited. Nat. Immunol. 4:303-304.

40. Rudensky, A.Y., Gavin, M., and Zheng, Y. 2006. FOXP3 and NFAT: partners in tolerance. Cell. 126:253-256.

41. Sakaguchi, S., Sakaguchi, N., Asano, M., and Toda, M. 1995. Immunologic self-tolerance maintained by activated T cells expressing IL- 2 receptor alphachains (CD25). Breakdown of a single mechanism of self-tolerance causes various autoimmune diseases. J. Immunol. 155:115-1164.

42. Bach, J.F. 2003. Regulatory T cells under scrutiny. Nat. Rev. Immunol. 3:189-198.

43. Shevach, E.M. 2002. CD4+ CD25+ suppressor T cells: more questions than answers. Nat. Rev. Immunol. 2:389-400.

44. Marson, A., et al. 2007. Foxp3 occupancy and regulation of key target genes during T-cell stimulation. Nature. 445:931-935.

45. Zheng, Y., et al. 2007. Genome-wide analysis of Foxp3 target genes in developing and mature regulatory T cells. Nature. 445:936-940.

46. Gavin, M.A., et al. 2007. Foxp3-dependent programme of regulatory T-cell differentiation. Nature. 445:771-775.

47. Wan, Y.Y., and Flavell, R.A. 2007. Regulatory T-cell functions are subverted and converted owing to attenuated Foxp3 expression. Nature. 445:766-770.

48. Bursuker, I., and North, R.J. 1984. Generation and decay of the immune response to a progressive fibrosarcoma. II. Failure to demonstrate postexcision immunity after the onset of $\mathrm{T}$ cell-mediated suppression of immunity. J. Exp. Med. 159:1312-1321.

49. Shimizu, J., Yamazaki, S., and Sakaguchi, S 1999. Induction of tumor immunity by removing CD25+CD4+ T cells: a common basis between tumor immunity and autoimmunity. J. Immunol. 163:5211-5218.

50. Tanaka, H., Tanaka, J., Kjaergaard, J., and Shu, S. 2002. Depletion of CD4+ CD25+ regulatory cells augments the generation of specific immune $\mathrm{T}$ cells in tumor-draining lymph nodes. J. Immunother. 25:207-217.

51. Steitz, J., Bruck, J., Lenz, J., Knop, J., and Tuting, T. 2001. Depletion of CD25(+) CD4(+) T cells and treatment with tyrosinase-related protein 2 -transduced dendritic cells enhance the interferon alphainduced, CD8(+) T-cell-dependent immune defense of B16 melanoma. Cancer Res. 61:8643-8646.

52. Sutmuller, R.P., et al. 2001. Synergism of cytotoxic $\mathrm{T}$ lymphocyte-associated antigen 4 blockade and depletion of CD25(+) regulatory $\mathrm{T}$ cells in antitumor therapy reveals alternative pathways for suppression of autoreactive cytotoxic T lymphocyte responses. J. Exp. Med. 194:823-832.

53. Grauer, O.M., et al. 2007. CD4+FoxP3+ regulatory $\mathrm{T}$ cells gradually accumulate in gliomas during tumor growth and efficiently suppress antiglioma immune responses in vivo. Int. J. Cancer. doi:10.1002/ijc.22607.

54. Chen, M.L., et al. 2005. Regulatory T cells suppress tumor-specific CD8 T cell cytotoxicity through TGF-beta signals in vivo. Proc. Natl. Acad. Sci. U. S. A. 102:419-424.

55. Nishikawa, H., Jager, E., Ritter, G., Old, L.J., and Gnjatic, S. 2005. CD4+ CD25+ regulatory T cells control the induction of antigen-specific CD4+ helper $\mathrm{T}$ cell responses in cancer patients. Blood. 106:1008-1011.

56. Levings, M.K., Sangregorio, R., and Roncarolo, M.G. 2001. Human $\mathrm{CD} 25^{+} \mathrm{CD} 4^{+} \mathrm{T}$ regulatory cells suppress naive and memory $T$ cell proliferation and can be expanded in vitro without loss of function. J. Exp. Med. 193:1295-1302.

57. Wang, R.F. 2006. Immune suppression by tumorspecific CD4(+) regulatory T-cells in cancer. Semin. Cancer Biol. 16:73-79.

58. Wang, R.F., Peng, G., and Wang, H.Y. 2006. Regulatory $T$ cells and Toll-like receptors in tumor immunity. Semin. Immunol. 18:136-142.

59. Woo, E.Y., et al. 2001. Regulatory CD4(+)CD25(+) $\mathrm{T}$ cells in tumors from patients with early- stage non-small cell lung cancer and late-stage ovarian cancer. Cancer Res. 61:4766-4772.

60. Curiel, T.J., et al. 2004. Specific recruitment of regulatory $\mathrm{T}$ cells in ovarian carcinoma fosters immune privilege and predicts reduced survival. Nat. Med. 10:942-949.

61. Woo, E.Y., et al. 2002. Regulatory T cells from lung cancer patients directly inhibit autologous $\mathrm{T}$ cell proliferation. J. Immunol. 168:4272-4276.

62. Javia, L.R., and Rosenberg, S.A. 2003. CD4+CD25+ suppressor lymphocytes in the circulation of patients immunized against melanoma antigens. J. Immunother. 26:85-93.

63. Somasundaram, R., et al. 2002. Inhibition of cytolytic $\mathrm{T}$ lymphocyte proliferation by autologous $\mathrm{CD} 4+/ \mathrm{CD} 25+$ regulatory $\mathrm{T}$ cells in a colorectal carcinoma patient is mediated by transforming growth factor-beta. Cancer Res. 62:5267-5272.

64. Wolf, A.M., et al. 2003. Increase of regulatory T cells in the peripheral blood of cancer patients. Clin. Cancer Res. 9:606-612

65. Sasada, T., Kimura, M., Yoshida, Y., Kanai, M., and Takabayashi, A. 2003. CD4+CD25+ regulatory T cells in patients with gastrointestinal malignancies: possible involvement of regulatory $\mathrm{T}$ cells in disease progression. Cancer. 98:1089-1099.

66. Liyanage, U.K., et al. 2002. Prevalence of regulatory $\mathrm{T}$ cells is increased in peripheral blood and tumor microenvironment of patients with pancreas or breast adenocarcinoma. J. Immunol. 169:2756-2761.

67. Matsuura, K., et al. 2006. Maturation of dendritic cells and T-cell responses in sentinel lymph nodes from patients with breast carcinoma. Cancer. 106:1227-1236.

68. Yang, Z.Z., Novak, A.J., Stenson, M.J., Witzig, T.E., and Ansell, S.M. 2006. Intratumoral CD4+CD25+ regulatory T-cell-mediated suppression of infiltrating CD4+ T cells in B-cell non-Hodgkin lymphoma. Blood. 107:3639-3646.

69. Chen, A., Liu, S., Park, D., Kang, Y., and Zheng, G. 2007. Depleting intratumoral CD4+CD25+ regulatory $\mathrm{T}$ cells via FasL protein transfer enhances the therapeutic efficacy of adoptive $\mathrm{T}$ cell transfer. Cancer Res. 67:1291-1298.

70. Carreras, J., et al. 2006. High numbers of tumorinfiltrating FOXP3-positive regulatory $\mathrm{T}$ cells are associated with improved overall survival in follicular lymphoma. Blood. 108:2957-2964.

71. Alvaro, T., et al. 2005. Outcome in Hodgkin's lymphoma can be predicted from the presence of accompanying cytotoxic and regulatory $\mathrm{T}$ cells. Clin. Cancer Res. 11:1467-1473.

72. Tiemessen, M.M., et al. 2006. Lack of suppressive $\mathrm{CD} 4+\mathrm{CD} 25+\mathrm{FOXP} 3+\mathrm{T}$ cells in advanced stages of primary cutaneous T-cell lymphoma. J. Invest. Dermatol. 126:2217-2223.

73. Bluestone, J.A., and Abbas, A.K. 2003. Natural versus adaptive regulatory T cells. Nat. Rev. Immunol. 3:253-257.

74. Larmonier, N., et al. 2007. Tumor-derived $\mathrm{CD} 4(+) \mathrm{CD} 25(+)$ regulatory $\mathrm{T}$ cell suppression of dendritic cell function involves TGF-beta and IL-10. Cancer Immunol. Immunother. 56:48-59.

75. Curiel, T.J., et al. 2003. Blockade of B7-H1 improves myeloid dendritic cell-mediated antitumor immunity. Nat. Med. 9:562-567.

76. Zou, W. 2006. Regulatory T cells, tumour immunity and immunotherapy. Nat. Rev. Immunol. 6:295-307.

77. Wang, H.Y., et al. 2004. Tumor-specific human CD4+ regulatory $\mathrm{T}$ cells and their ligands: implications for immunotherapy. Immunity. 20:107-118.

78. Ghiringhelli, F., Menard, C., Martin, F., and Zitvogel, L. 2006. The role of regulatory T cells in the control of natural killer cells: relevance during tumor progression. Immunol. Rev. 214:229-238.

79. Lim, H.W., Hillsamer, P., Banham, A.H., and Kim, C.H. 2005. Cutting edge: direct suppression of B cells by CD $4+\mathrm{CD} 25+$ regulatory T cells. J. Immunol. 175:4180-4183.

80. Hori, S., Nomura, T., and Sakaguchi, S. 2003. Control of regulatory $\mathrm{T}$ cell development by the transcription factor foxp3. Science. 299:1057-1061.

81. Fontenot, J.D., Gavin, M.A., and Rudensky, A.Y. 2003. Foxp3 programs the development and function of CD $4+\mathrm{CD} 25+$ regulatory T cells. Nat. Immunol. 4:330-336.

82. Curiel, T.J. 2007. Regulatory T cell development: is Foxp3 the decider? Nat. Med. 13:250-253.

83. Graca, L. 2005. New tools to identify regulatory $T$ cells. Eur. J. Immunol. 35:1678-1680.

84. Roncador, G., et al. 2005. Analysis of FOXP3 protein expression in human CD4+CD25+ regulatory $\mathrm{T}$ cells at the single-cell level. Eur. J. Immunol. 35:1681-1691.

85. Fontenot, J.D., et al. 2005. Regulatory T cell lineage specification by the forkhead transcription factor foxp3. Immunity. 22:329-341.

86. Wan, Y.Y., and Flavell, R.A. 2005. Identifying Foxp3-expressing suppressor $\mathrm{T}$ cells with a bicistronic reporter. Proc. Natl. Acad. Sci. U. S. A. 102:5126-5131.

87. Knutson, K., Curiel, T., Salazar, L., and Disis, M. 2003. Immunologic principles and immunotherapeutic approaches in ovarian cancer. Hematol. Oncol. Clin. North Am. 17:1051-1073.

88. Zou, W., et al. 2001. Stromal-derived factor-1 in human tumors recruits and alters the function of plasmacytoid precursor dendritic cells. Nat. Med. 7:1339-1346.

89. Wolf, D., et al. 2005. The expression of the regulatory $\mathrm{T}$ cell-specific forkhead box transcription factor FoxP3 is associated with poor prognosis in ovarian cancer. Clin. Cancer Res. 11:8326-8331.

90. Bates, G.J., et al. 2006. Quantification of regulatory $T$ cells enables the identification of high-risk breast cancer patients and those at risk of late relapse. J. Clin. Oncol. 24:5373-5380.

91. Kobayashi, N., et al. 2007. FOXP3+ regulatory T cells affect the development and progression of hepatocarcinogenesis. Clin. Cancer Res. 13:902-911.

92. Prabhala, R.H., et al. 2006. Dysfunctional T regulatory cells in multiple myeloma. Blood. 107:301-304.

93. Foss, F.M. 2000. DAB(389)IL-2 (ONTAK): a novel fusion toxin therapy for lymphoma. Clin. Lymphoma. 1:110-116; discussion 117.

94. Barnett, B., Kryczek, I., Cheng, P., Zou, W., and Curiel, T.J. 2005. Regulatory $\mathrm{T}$ cells in ovarian cancer: biology and therapeutic potential. Am.J. Reprod. Immunol. 54:369-377.

95. Knutson, K.L., et al. 2006. IL-2 immunotoxin therapy modulates tumor-associated regulatory $\mathrm{T}$ cells and leads to lasting immune-mediated rejection of breast cancers in neu-transgenic mice. J. Immunol. 177:84-91.

96. Dannull, J., et al. 2005. Enhancement of vaccinemediated antitumor immunity in cancer patients after depletion of regulatory T cells. J. Clin. Invest. 115:3623-3633. doi:10.1172/JCI25947. 
97. Chesney, J., Rasku, M., Clem, A., and Miller, D. 2006. Denileukin diftitox depletes T regulatory cells and causes regression of melanoma metastases in humans [abstract]. European Journal of Cancer Supplements. 4:84

98. Mahnke, K., et al. 2007. Depletion of $\mathrm{CD}^{+}{ }^{+} \mathrm{CD} 25^{+}$ human regulatory $\mathrm{T}$ cells in vivo: kinetics of Treg depletion and alterations in immune functions in vivo and in vitro. Int. J. Cancer. doi:10.1002/ijc.22617.

99. Attia, P., Maker, A.V., Haworth, L.R., Rogers-Freezer, L., and Rosenberg, S.A. 2005. Inability of a fusion protein of IL-2 and diphtheria toxin (Denileukin Diftitox, DAB389IL-2, ONTAK) to eliminate regulatory $\mathrm{T}$ lymphocytes in patients with melanoma. J. Immunother. 28:582-592.

100.Jarnicki, A.G., Lysaght, J., Todryk, S., and Mills, K.H. 2006. Suppression of antitumor immunity by IL-10 and TGF-beta-producing T cells infiltrating the growing tumor: influence of tumor environment on the induction of CD4+ and CD8+ regulatory T cells. J. Immunol. 177:896-904.

101. Rüter, J., et al. 2007. Altering regulatory T cell function in cancer immunotherapy: a novel means to boost efficacy. Front. Biosci. In press.

102.Li, B., et al. 2006. Vascular endothelial growth factor blockade reduces intratumoral regulatory $\mathrm{T}$ cells and enhances the efficacy of a GM-CSFsecreting cancer immunotherapy. Clin. Cancer Res. 12:6808-6816.

103.Zhou, G., Drake, C.G., and Levitsky, H.I. 2006. Amplification of tumor-specific regulatory $\mathrm{T}$ cells following therapeutic cancer vaccines. Blood. 107:628-636

104. Banerjee, D.K., et al. 2006. Expansion of FOXP3high regulatory $T$ cells by human dendritic cells (DCs) in vitro and after injection of cytokine-matured DCs in myeloma patients. Blood. 108:2655-2661.

105.Ahmadzadeh, M., and Rosenberg, S.A. 2006 IL-2 administration increases CD4+ CD25(hi) Foxp3+ regulatory $\mathrm{T}$ cells in cancer patients. Blood. 107:2409-2414.

106.Berek, J.S., Schultes, B.C., and Nicodemus, C.F. 2003. Biologic and immunologic therapies for ovarian cancer. J. Clin. Oncol. 21:168-174.

107. Windbichler, G.H., et al. 2000. Interferon-gamma in the first-line therapy of ovarian cancer: a randomized phase III trial. Br. J. Cancer. 82:1138-1144.

108. Rongcun, Y., et al. 1998. Interferon gamma impairs the ability of monocyte-derived dendritic cells to present tumour-specific and allo-specific antigens and reduces their expression of CD1A, CD80 and CD4. Cytokine. 10:747-755.

109. Kudo-Saito, C., Schlom, J., Camphausen, K., Coleman, C.N., and Hodge, J.W. 2005. The requirement of multimodal therapy (vaccine, local tumor radiation, and reduction of suppressor cells) to eliminate established tumors. Clin. Cancer Res. 11:4533-4544.

110.Taieb, J., et al. 2006. Chemoimmunotherapy of tumors: cyclophosphamide synergizes with exosome based vaccines. J. Immunol. 176:2722-2729.

111.Nair, S., Boczkowski, D., Fassnacht, M., Pisetsky, D., and Gilboa, E. 2007. Vaccination against the forkhead family transcription factor Foxp3 enhances tumor immunity. Cancer Res. 67:371-380.

112. Wei, S., et al. 2005. Plasmacytoid dendritic cells induce $\mathrm{CD}^{+}$regulatory $\mathrm{T}$ cells in human ovarian carcinoma. Cancer Res. 65:5020-5026.

113.Taniguchi, M., Harada, M., Kojo, S., Nakayama, T., and Wakao, H. 2003. The regulatory role of Valpha14 NKT cells in innate and acquired immune response. Annu. Rev. Immunol. 21:483-513.

114.Almand, B., et al. 2001. Increased production of immature myeloid cells in cancer patients: a mechanism of immunosuppression in cancer. J. Immunol. 166:678-689.

115.Kusmartsev, S., and Gabrilovich, D.I. 2006. Role of immature myeloid cells in mechanisms of immune evasion in cancer. Cancer Immunol. Immunother. 55:237-245.

116. Kryczek, I., et al. 2006. B7-H4 expression identifies a novel suppressive macrophage population in human ovarian carcinoma. J. Exp. Med. 203:871-881.

117. Terabe, M., et al. 2005. A nonclassical non-Valpha14Jalpha18 CD1d-restricted (type II) NKT cell is sufficient for down-regulation of tumor immunosurveillance. J. Exp. Med. 202:1627-1633.

118. Mahic, M., Yaqub, S., Johansson, C.C., Tasken, K., and Aandahl, E.M. 2006. FOXP3+CD4+CD25+ adaptive regulatory $\mathrm{T}$ cells express cyclooxygenase- 2 and suppress effector $\mathrm{T}$ cells by a prostaglandin E2dependent mechanism. J. Immunol. 177:246-254.

119.Polanczyk, M.J., et al. 2004. Cutting edge: estrogen drives expansion of the $\mathrm{CD} 4+\mathrm{CD} 25+$ regulatory $\mathrm{T}$ cell compartment. J. Immunol. 173:2227-2230.

120.Tisdale, J.F., Dunn, D.E., and Maciejewski, J. 2000. Cyclophosphamide and other new agents for the treatment of severe aplastic anemia. Semin. Hematol. 37:102-109.

121.Berd, D., and Mastrangelo, M.J. 1987. Effect of low dose cyclophosphamide on the immune system of cancer patients: reduction of T-suppressor function without depletion of the CD8+ subset. Cancer Res. 47:3317-3321.

122. Ghiringhelli, F., et al. 2007. Metronomic cyclophosphamide regimen selectively depletes CD4(+)CD25 $(+)$ regulatory $\mathrm{T}$ cells and restores $\mathrm{T}$ and $\mathrm{NK}$ effector functions in end stage cancer patients. Cancer Immunol. Immunother. 56:641-648. 